\title{
Analysis of perioperative pain management in vascular surgery indicates that practice does not adhere with guidelines: a retrospective cross-sectional study
}

\author{
This article was published in the following Dove Press journal: \\ Journal of Pain Research \\ 17 January 2017 \\ Number of times this article has been viewed
}

\section{Krste Boric' \\ Matija Boric ${ }^{1,2}$ \\ Teo Boric ${ }^{3}$ \\ Livia Puljak'}

'Laboratory for Pain Research, University of Split School of Medicine, Split, Croatia; ${ }^{2}$ Department of Abdominal Surgery, ${ }^{3}$ Department of Vascular Surgery, University Hospital Split, Split, Croatia
Correspondence: Livia Puljak Laboratory for Pain Research, University of Split School of Medicine, Soltanska 2, 21000 Split, Croatia

$\mathrm{Tel}+38521557807$

Fax +385 2I 557811

Email livia@mefst.hr
Background: Inadequate treatment of pain related to surgery may be associated with complications and prolonged recovery time and increased morbidity and mortality rates. We investigated perioperative pain management in vascular surgery and compared it with the relevant guidelines for the treatment of perioperative pain.

Methods: We conducted a retrospective study on 501 patients who underwent vascular surgery at the University Hospital Split, Croatia. We collected the following data from patients' charts: age, gender, premedication, preoperative patient's physical status, type of surgery, duration of surgery and anesthesia, type of anesthesia, postoperative analgesia, and need for intensive care. We examined departmental procedures to assess adherence to guidelines for perioperative pain management.

Results: None of the 501 patients' charts recorded information about perioperative pain intensity, $28 \%$ of patients did not receive any medication the night before their elective surgical procedures, and $17 \%$ of patients did not receive premedication immediately before the procedure. Most patients $(66 \%)$ did not receive any pain medication in the operating room after surgery. Following surgery, $36 \%$ of patients were monitored in the intensive care units, while the rest were released to the ward. Some patients $(17 \%)$ did not receive any analgesia after surgery. Procedures at the department did not adhere to the current recommendations for perioperative pain management.

Conclusion: The study indicates that management of surgery-related pain in complex vascular procedures at this hospital did not follow guidelines for the management of acute perioperative pain. Our finding that most patients did not receive appropriate analgesia after vascular surgery leads to the conclusion that the institution would benefit from developing guidelines for the management of acute perioperative pain, which should be applied in all cases.

Keywords: perioperative pain, postoperative pain, vascular surgery, pain management, anesthesia, analgesia

\section{Introduction}

The American Society of Anesthesiologists (ASA) guidelines for the treatment of pain in the perioperative period define postoperative acute pain as pain occurring in surgical patients following a procedure. ${ }^{1}$ Every year, over 234 million surgical procedures are performed globally. Therefore, appropriate pain control after surgery 
is of great importance. ${ }^{2}$ Research has shown that acute postoperative pain occurs in $80 \%$ of patients, with $86 \%$ of these experiencing moderate to severe pain. ${ }^{3}$ Inadequate treatment of postoperative pain may affect the development of complications and prolonged recovery time and may increase morbidity and mortality. ${ }^{4-6}$ Appropriate pain management contributes to a shorter duration of hospitalization, lower hospital costs, and increased levels of patient satisfaction. The World Health Organization and International Association for the Study of Pain consider treatment of pain as a basic human right, so it is not surprising that the treatment of postoperative pain is used as a measure of the quality of hospital practice. ${ }^{7-9}$

After recovery from anesthesia, pain management should be based on the patient's self-report to assess pain perceptions and cognitive response. A patient self-reporting of pain has been identified as the single most reliable indicator of the existence and intensity of acute pain and any concomitant affective discomfort or distress. ${ }^{10}$ The pain management cycle includes not only assessment but also intervention and reassessment; these three comprise the cycle that has been a measure of the quality of care for some years. ${ }^{11}$ However, it is unclear how widely those concepts are applied in vascular surgery perioperative care.

In 2012, the ASA published the latest version of its guidelines for the treatment of acute pain in the perioperative period. ${ }^{1}$ Adherence to these guidelines within the field of vascular surgery has been little studied. Therefore, the aim of this study was to assess the types of anesthesia and analgesia given perioperatively in serious vascular surgical procedures and to determine whether perioperative procedures adhered to the guidelines. Our main specific objectives were to analyze the number of patients who did not receive any premedication before surgery or analgesia after surgery, the types of analgesia that were provided to patients and its frequency, and the level of compliance with the relevant guidelines.

\section{Methods}

\section{Participants}

We conducted a retrospective, cross-sectional study among patients who underwent vascular surgery in one institution, the University Hospital Split, Croatia, at the Department of Surgery, Division of Vascular Surgery during 2013.

We used the following inclusion criteria: patients who underwent vascular surgery, which included limb amputations, vascular bypass, varicectomy, embolectomy, aortofemoral thromboendarterectomy, creation of arteriovenous fistula for hemodialysis, carotid endarterectomy. Patients younger than 18 years or whose medical charts were unavailable were excluded.

\section{Ethics}

The study was approved by the Ethics Committee of the University Hospital Split (Class 500-003/15-01/03, Ur. br.: 2181-147-01/06/JB-13-2). The Ethics Committee waived the need for patient consent owing to retrospective data collection.

\section{Study protocol}

We analyzed types of anesthesia and analgesia during and after procedures in vascular surgery and perioperative procedures. We compared the collected data with the current ASA guidelines for perioperative pain. ${ }^{1}$ Data were extracted from physical patient charts. One author extracted the data. We used a formalized data collection instrument with a manual defining variables and abstraction procedures. We conducted formal training for data extraction and piloted data collection instrument before proceeding with data collection.

We retrieved patients' charts from the hospital archives and extracted the following data: age, gender, type and dosage of premedication, preoperative physical status according to the ASA classification, ${ }^{12}$ type of surgery, duration of surgery - surgical and anesthesia time, anesthesia type and dosage, postoperative analgesia type and dosage for each postoperative day, and the need for intensive care.

We followed the Summary of Recommendations from the ASA guidelines for the treatment of pain in the perioperative period to examine if the perioperative treatment conformed with the guidelines. ${ }^{1}$ Based on these recommendations, we recorded the following information: whether the anesthesiologists provided training for healthcare workers involved in perioperative care of patients who underwent surgery in the department; whether pain intensity was evaluated before the operation and was recorded in the patient's medical chart; whether any information about the assessment of pain using standardized instruments was recorded in the medical charts; whether the intensity of pain was documented, whether any effect of treatment was mentioned, as well as side effects of therapy for pain; whether any multimodal measures for pain management were used (ie, use of at least two analgesics with different modes of action); whether acetaminophen was used for pain management; whether patients were placed in a separate room for postoperative recovery after surgery; and whether there was intensive monitoring of patients after surgery. 


\section{Raw data}

Raw data generated in the study are available in the open access repository Figshare (DOI: 10.6084/m9.figshare.3510233; URL: https://figshare.com/s/790b362b29fb178c47ef).

\section{Statistical analysis}

We based our sample size calculation on the assumption that $40 \%$ of patients would not receive an analgesic after surgery. To obtain $95 \%$ statistical power, with an alpha value of 0.05 , we calculated that 130 patients were necessary in each group. To compensate for potential missing data in retrospective chart review, we included all 501 patients who underwent vascular surgery during 1 year.

We coded data sets for each patient, anonymized them and extracted data into electronic datasheets. We assessed normality of data distribution using the Kolmogorov-Smirnov test. We presented data as mean and standard deviation $(\mathrm{M} \pm$ SD). We analyzed differences between different groups using $t$-tests or the Mann-Whitney test, depending on the normality of data distribution. Association between variables was analyzed using Spearman's coefficient of correlation. Missing data were reported in descriptive statistics, where relevant. We used GraphPad Prism statistical software for data analysis (GraphPad Software, La Jolla, CA, USA).

\section{Results}

\section{Participants}

In the analyzed population of 501 patients who underwent vascular surgery during 2013, majority were men. The average age was 67 years (range: 28-96 years). The majority of patients had ASA status, ${ }^{2}$ indicating patients with mild or systemic diseases (Table 1). Patients usually received spinal or local anesthesia (Table 1).

\section{The intensity of pain}

Detailed examination of the patients' charts revealed that information about pain intensity was not recorded for the analyzed patients.

\section{Monitoring after surgery}

Following surgery, 179 (36\%) patients were admitted to the intensive care unit, while the remaining 322 (64\%) returned to a regular ward. The average duration of intensive care was 13.5 hours.

\section{Perioperative treatment}

\section{Analgesia administered before surgery}

Prior to surgery, 38 patients received analgesics. Excluding emergency procedures in which the patients were not able to
Table I Characteristics of the study participants

\begin{tabular}{ll}
\hline Variable & \\
\hline Gender, N (\%) & $310(6 \mathrm{I})$ \\
Men & $191(39)$ \\
Women & $66.9 \pm 14.08$ \\
Age, $M \pm$ SD & \\
ASA, N (\%) & $40(10)$ \\
I: A normal healthy patient & $5(1.3)$ \\
I/2: & $143(37)$ \\
2: A patient with mild systemic disease & $42(11)$ \\
2/3: & $132(34)$ \\
3: A patient with severe systemic disease & $18(4.6)$ \\
3/4: & $8(2)$ \\
4: A patient with severe systemic disease that is a & \\
constant threat to life & $113(23)$ \\
Missing data & \\
Type of anesthesia, N (\%) & $3(0.6)$ \\
Missing data & $4 I(8.1)$ \\
Local & $305(6 \mathrm{I})$ \\
General & $139(28)$ \\
Spinal & $13(2.7)$ \\
Combined general and spinal &
\end{tabular}

Abbreviations: M, mean; SD, standard deviation; ASA, American Society of Anesthesiologists.

receive preoperative analgesia, only 38 patients (11\%) undergoing elective surgery received analgesics before surgery.

\section{Therapy before surgery}

We found that 247 patients $(49 \%)$ did not receive any treatment the night before surgery, while the remaining 254 $(51 \%)$ received a variety of drugs, of which the most common was diazepam. Among patients undergoing elective surgery $(\mathrm{N}=351)$, the proportion who received therapy the night before their surgery was $72 \%$. Records indicated that 209 patients $(41 \%)$ did not receive premedication before their surgery, while others received diazepam, alprazolam, or midazolam. Among patients who underwent elective surgery, therapy on the day of surgery was given to 290 (83\%) (Table 2).

\section{Pharmacological treatment in the operating room} During surgery, 160 patients (32\%) received medication other than an anesthetic, most often atropine, metamizole sodium, and ephedrine. After the operation, when they were still in the operating room, 169 patients $(34 \%)$ received medication, most often atropine or neostigmine, and $90 \%$ received more than one medication. After the operation, 332 patients $(66 \%)$ did not receive any medication.

\section{Analgesic therapy after surgery}

Following their return to the Department of Vascular Surgery for recovery, 85 patients (17\%) did not receive any analgesia 
Table 2 Preoperative therapy

\begin{tabular}{ll}
\hline Therapy the night before surgery & $\mathbf{N}(\%)$ \\
\hline None & $97(28)$ \\
Received medication & $254(72)$ \\
Diazepam, $5 \mathrm{mg}$ & $25 \mathrm{I}(99)$ \\
Diazepam, $10 \mathrm{mg}$ & $2(0.8)$ \\
Alprazolam, I mg & $1(0.4)$ \\
\hline Premedication & $\mathbf{N}(\%)$ \\
\hline None & $61(17)$ \\
Received medication & $290(83)$ \\
Diazepam, $5 \mathrm{mg}$ & $217(74)$ \\
Diazepam, I0 mg & $32(1 \mathrm{I})$ \\
Midazolam, 7.5 mg & $41(14)$ \\
Midazolam, $2 \mathrm{mg}$ & $\mathrm{I}(0.4)$ \\
Alprazolam, I mg & $\mathrm{I}(0.4)$ \\
\hline
\end{tabular}

Table 3 Postoperative therapy (some patients received multiple drugs)

\begin{tabular}{ll}
\hline Drugs received in the operating room & N (\%) \\
\hline Without drugs & $332(66)$ \\
With drugs & $169(34)$ \\
Atropine & $152(90)$ \\
Neostigmine & $148(88)$ \\
Diklofekan & $13(7.6)$ \\
Metamizole sodium & $10(6)$ \\
Tramadol & $1(0,6)$ \\
Chirocaine, fentanyl & I $(0.6)$ \\
\hline Postoperative analgesia at the department & $\mathbf{N}(\%)$ \\
\hline Without postoperative analgesia & $85(17)$ \\
With postoperative analgesia & $416(83)$ \\
Metamizole sodium alone & $241(58)$ \\
Metamizole sodium combined with other drugs & $98(23)$ \\
Diklofenak & $23(5.5)$ \\
Diklofenak combined with other drugs & $33(8)$ \\
Tramadol & II (2.6) \\
Tramadol combined with other drugs & $93(22)$ \\
Morphine alone and combined with other drugs & $4(1)$ \\
Ibuprofen & $4(1)$ \\
Ibuprofen combined with other drugs & $10(2.3)$ \\
Pethidine alone and combined with other drugs & $4(1)$ \\
Epidural catheter (chirocaine, lidocaine, fentanyl) & $11(2.6)$ \\
\hline
\end{tabular}

until discharge from the hospital. Patients who did receive an analgesic most commonly received metamizole sodium either alone or in combination with another analgesic. Of the 85 patients who did not receive analgesia after surgery, $50(59 \%)$ of them underwent varicectomy. Of the 416 patients who received postoperative analgesics in the department, $214(51 \%)$ had received one analgesic or more but on the first day only (ie, on the day of surgery), and 125 patients $(30 \%)$ received only one dose of an analgesic (Table 3 ).

\section{Surgical procedures and analgesics}

Among the 501 operations, 351 (70\%) were elective and $150(30 \%)$ were emergency procedures. The most common type of surgery during the study period was varicectomy $(29 \%)$. The average duration of all procedures was $77.5 \pm 47.8$ minutes. The operating time varied for different types of surgery. Vascular bypass procedures (166 $\pm 60.3 \mathrm{~min}-$ utes) involved the longest operating times (Table 4).

Duration of surgical procedure was significantly longer in patients who received analgesia than in those who did not receive an analgesic ( $81 \pm 49$ minutes vs $59 \pm 39$ minutes; Mann-Whitney $U=1028, p<0.001)$. A significant but weak positive correlation was found between the duration of surgery and the likelihood of receiving analgesic (Spearman $r=0.236, p<0.001$ ). There was also a weak significant correlation between age and receiving analgesics; older patients more often received analgesics (Spearman $r=0.194, p<0.001$ ). There was no association between patient sex and receiving analgesics (Spearman $r=-0.039, p=0.379$ ).

\section{Comparing practice with guidelines}

We compared published guidelines for acute pain management in the perioperative setting with practice in the analyzed setting and found weaknesses in all domains of the guidelines. The hospital did not have institutional guidelines/protocols for pain management, and therefore pain management was purely clinician guided. Anesthesiologists did not provide any organized education for physicians or nurses involved in perioperative care. Healthcare workers did not record perioperative pain intensity on patients' medical charts. Information about pain therapy only occurred when such therapy was prescribed. However, we did not find information about adverse events of analgesia in a single patient chart. Multimodal measures for pain management were not used, and acetaminophen was very rarely used as a postoperative analgesic. There was no room dedicated for postoperative recovery, and patients did not receive intensive monitoring after surgery unless their condition was life threatening and they therefore needed intensive care.

\section{Discussion}

Our results show that postoperative pain management following complex vascular operations was inadequate and pain intensity was not recorded in patients' charts in our hospital. Postoperative pain is one of the most commonly reported symptoms after surgery. ${ }^{13}$ Although many effective interventions are available for the treatment of acute pain, most surgical patients experience acute, often intense, postoperative pain. ${ }^{3}$ Studies have shown that $80 \%$ of patients experience acute postoperative pain, and $86 \%$ of them rate the pain as moderate to severe. ${ }^{3}$ Some surgical patients 
Table 4 Type, duration, and postoperative analgesia of different surgical procedures

\begin{tabular}{|c|c|c|c|c|}
\hline Type of surgery & $\begin{array}{l}\text { Frequency, } \\
\text { N (\%) }\end{array}$ & $\begin{array}{l}\text { Operating time } \\
\text { in } \min , M \pm S D\end{array}$ & $\begin{array}{l}\text { Anesthesia time } \\
\text { in } \mathbf{m i n}, \mathbf{M} \pm \mathrm{SD}\end{array}$ & $\begin{array}{l}\text { Postoperative } \\
\text { analgesia, N (\%) }\end{array}$ \\
\hline Amputations & $31(6.2)$ & $47.4(13)$ & $72.2(16)$ & $28(90)$ \\
\hline Vascular bypass & $47(9.3)$ & $166(60)$ & $212(71)$ & $46(98)$ \\
\hline Varicectomy & $147(29)$ & $49.4(16)$ & $76.2(20)$ & $97(66)$ \\
\hline Embolectomy & $4(0.8)$ & $71.6(10)$ & $101.6(10)$ & $4(100)$ \\
\hline Femoral artery thromboendarterectomy & $22(4.4)$ & II $2.2(55)$ & $145(57)$ & $20(91)$ \\
\hline Arteriovenous fistula & $2(0.4)$ & - & - & $0(0)$ \\
\hline Aortofemoral thromboendarterectomy & $98(20)$ & $100.4(25)$ & $135.4(30)$ & $96(98)$ \\
\hline Emergency amputations & $114(23)$ & $48.7(18)$ & $68.3(16)$ & $97(85)$ \\
\hline Emergency vascular bypass & $\mathrm{I}(0.2)$ & - & - & $0(0)$ \\
\hline Emergency embolectomy & $30(6)$ & $73.3(26)$ & $99.6(38)$ & $24(80)$ \\
\hline Emergency aortofemoral thromboendarterectomy & $5(I)$ & $88(28)$ & $110(24)$ & $4(80)$ \\
\hline Total/average & $501(100)$ & $77.5(48)$ & $105.8(56)$ & $501(100)$ \\
\hline
\end{tabular}

Abbreviations: $M$, mean; SD, standard deviation.

experience postoperative pain that lasts for weeks, months, or even years after surgery - much longer than the usual period necessary for tissue healing. ${ }^{14}$ Not only is pain an unpleasant experience but it also increases the occurrence of complications and affects the outcome of treatment. ${ }^{4-6}$ Today, it is considered that the appropriate treatment of pain is a basic human right. ${ }^{9}$

In our study population, the majority of patients received an anxiolytic the night before surgery and on the day of the operation. However, Beydon et $\mathrm{al}^{15}$ consider that giving anxiolytics before surgery is not more effective than placebo. Research among patients who received general anesthesia and were given lorazepam, placebo or no premedication, showed that premedication with lorazepam did not improve the experience of patients, but was associated with a longer time for extubation and a lower rate of early cognitive recovery. This research casts doubt on the routine use of lorazepam for premedication in patients undergoing general anesthesia. ${ }^{16}$ However, Scavée et $\mathrm{al}^{17}$ investigated the impact of fear and anxiety on the outcome of vascular surgery and suggested that fear and anxiety increase the incidence of postoperative complications. Therefore, anxiolytics may have long-term beneficial effects.

Among patients who received some analgesia in the department, most received only metamizole sodium, although the current guidelines recommend multimodal treatment. ${ }^{18}$ In our study, $26 \%$ of patients received an opioid analgesic alone or in combination with another analgesic, usually metamizole sodium. None of the 501 patients received the combination of acetaminophen and nonsteroidal anti-inflammatory drugs, although it has been shown that this combination works better than either drug alone. ${ }^{19}$

Although epidural analgesia is considered the best method of postoperative analgesia for complex vascular surgery, ${ }^{20}$ it was used in only $2.6 \%$ of the patients. Studies have shown that epidural analgesia reduces cardiac and pulmonary complications, length of stay in the intensive care unit, and the time of hospital discharge after elective surgery for aortic aneurysms. ${ }^{21}$ Furthermore, although according to the Karanikolas et $\mathrm{al}^{22}$ the best way of preventing the development of chronic phantom pain after amputation is the use of epidural analgesia or patient-controlled analgesia, starting 48 hours before surgery and continued for 48 hours after the procedure, none of the patients who underwent limb amputation in our study period received epidural analgesia. Following the operation in most hospitals, patients are placed in a room for postoperative monitoring, ie, a recovery room. Despite the large number of operations performed in the University Hospital in Split, it has no recovery room. As many as $64 \%$ of patients were returned to the Department of Vascular Surgery immediately after surgery. Katz and $\mathrm{Kohl}^{23}$ in their study showed that a combination of quality care conducted in the post-anesthesia care unit can greatly reduce the need for admission to the intensive care unit. Therefore, use of a dedicated recovery room should reduce the number of patients requiring intensive care and associated treatment costs.

Our study was retrospective, and therefore it is impossible to know whether patients asked staff for pain medications once they returned from the operating room to the ward, or whether the decision to provide analgesics depended entirely on the healthcare staff. Even when analgesics are administered, it is known that undertreatment of pain can result from inadequate or complete lack of repeated reassessment of pain by healthcare professionals. Patients should be asked repeatedly about their pain intensity and whether they need analgesia. A study conducted among nurses revealed that, of all patient-centered activities related to pain, only $4 \%$ of 
activities included repeated assessment of pain intensity after providing an analgesic. ${ }^{24}$ It is often necessary to reassess pain shortly after administering analgesia and, if necessary, to adjust the dose or type of analgesia. ${ }^{25}$

We also found a significant positive association between duration of the surgical procedure and the likelihood of receiving an analgesic. Thus, $98 \%$ of the patients who underwent vascular bypass surgery received an analgesic, while only $66 \%$ of patients received analgesia after varicectomy. These findings suggest that procedure-related factors may influence pain intensity and analgesic requirements, and this should be studied in more detail.

The fact that we found no data on pain intensity in the medical charts does not necessarily mean that medical staff in the department, including doctors and nurses, did not check the intensity of patients' pain. However, the fact that not all patients received analgesia after surgery suggests that staff probably did not assess pain intensity regularly.

We conclude that pain management should become a priority among healthcare staff involved in postoperative patient care in Croatia. Physicians are important in this process, but the role of nurses is also crucial because they can observe patients more often after the surgery, provide prescribed analgesics, and assess pain intensity regularly. Greater efforts should be invested in changing practice because it has been shown that there may be a discrepancy between what the healthcare workers say they did and what they actually did. ${ }^{26}$ The presence of anesthesiologists only in the operating room may be part of the problem because anesthesiologists need to coordinate postoperative therapy in the department with surgeons and nurses. One of the recommendations of guidelines for postoperative pain management is the availability of an anesthesiologist for 24 hours after surgery for consultation with nurses, surgeons, and other physicians involved in the treatment of patients regarding the treatment of perioperative pain. ${ }^{1}$ However, due to lack of staff, such recommendations are sometimes difficult to implement in the hospital environment.

All this contributes to the undesirable situation regarding pain management in the study setting. Suggestions for improving the treatment of pain include education of hospital staff, team coordination, communication with patients, and speed of action. ${ }^{27}$ In addition to postoperative analgesia, research has shown the inadequacy of premedication and therapy the night before a procedure. One important reason for inadequate analgesia is often the lack of medical staff, and this is one of the problems present in the analyzed institution and already described in the literature as one of the problems indicated by patients seeking pain treatment. ${ }^{28}$

In the same hospital, earlier research in another surgical department also showed that pain was not adequately managed: among patients who underwent complex ophthalmic surgical procedures, $46 \%$ of patients did not receive any analgesia after surgery, and among those who received analgesic, 98\% received only one dose of analgesics, and $93 \%$ of patients received analgesia only on the first postoperative day. ${ }^{29}$ This is the only previously published study about the treatment of acute postoperative pain in the analyzed hospital. However, these data indicate that the analyzed hospital would benefit from implementing guidelines for acute perioperative pain management that should be applied in all cases.

To the best of our knowledge, this is the first study analyzing the management of pain in patients undergoing vascular surgery. Limitations of the study include its retrospective, cross-sectional design, which relies on data present in patient charts, and lack of information about pain intensity. Additional limitations may be related to the extraction of data from patient charts, since numerous processes can introduce bias. ${ }^{30}$ Additionally, the study was conducted in only one country and in one institution. Therefore, this study may not represent the patterns of pain management in the specialty as a whole, but a number of previous studies in different fields have indicated insufficient pain management in surgical patients. Thus, we hope our findings will open a discussion about pain management in vascular surgery. Future studies on the subject should include prospective study designs and analyze pain intensity, patient satisfaction with analgesia, patient quality of life, and various factors that can impact pain in various vascular surgical procedures. Organization of work and attitudes of staff involved in provision of surgery, anesthesia, and other patient care should be examined to see what can be changed systematically to improve perioperative pain management.

\section{Conclusion}

Based on our findings, we conclude that perioperative pain management in vascular surgery and adherence to perioperative pain management guidelines are inadequate in some centers. The studied institution would benefit from developing guidelines for perioperative pain management, adjusted to local conditions.

\section{Acknowledgment}

The authors gratefully acknowledge the help of Dr. Elizabeth Wager of Sideview, for manuscript editing. 


\section{Disclosure}

The authors report no conflicts of interest in this work.

\section{References}

1. American Society of Anesthesiologists Task Force on Acute Pain M. Practice guidelines for acute pain management in the perioperative setting: an updated report by the American Society of Anesthesiologists Task Force on Acute Pain Management. Anesthesiology. 2012;116(2):248-273.

2. Weiser TG, Regenbogen SE, Thompson KD, et al. An estimation of the global volume of surgery: a modelling strategy based on available data. Lancet. 2008;372(9633):139-144.

3. Apfelbaum JL, Chen C, Mehta SS, Gan TJ. Postoperative pain experience: results from a national survey suggest postoperative pain continues to be undermanaged. Anesth Analg. 2003;97(2):534-540.

4. Sharrock NE, Cazan MG, Hargett MJ, Williams-Russo P, Wilson PD Jr. Changes in mortality after total hip and knee arthroplasty over a ten-year period. Anesth Analg. 1995;80(2):242-248.

5. Katz J, Jackson M, Kavanagh BP, Sandler AN. Acute pain after thoracic surgery predicts long-term post-thoracotomy pain. Clin J Pain. 1996;12(1):50-55.

6. Kehlet $\mathrm{H}$, Holte K. Effect of postoperative analgesia on surgical outcome. Br J Anaesth. 2001;87(1):62-72.

7. Practice guidelines for acute pain management in the perioperative setting. A report by the American Society of Anesthesiologists Task Force on Pain Management, Acute Pain Section. Anesthesiology. 1995;82(4):1071-1081.

8. Twersky R, Fishman D, Homel P. What happens after discharge? return hospital visits after ambulatory surgery. Anesth Analg. 1997;84(2):319-324.

9. Brennan F, Carr DB, Cousins M. Pain management: a fundamental human right. Anesth Analg. 2007;105(1):205-221.

10. The integrated approach to the management of pain. NIH Consensus Development Conference. J Pain Symptom Manag. 1987;2(1):35-44.

11. National Database of Nursing Quality Indicators (NDNQI), 2010. Available from: http://nursingandndnqi.weebly.com/ndnqi-indicators. html.

12. Daabiss M. American Society of Anaesthesiologists physical status classification. Indian J Anaesth. 2011;55(2):111-115.

13. Chung F, Un V, Su J. Postoperative symptoms 24 hours after ambulatory anaesthesia. Can J Anaesth. 1996;43(11):1121-1127.

14. Shipton EA. The transition from acute to chronic post surgical pain. Anaesth Intens Care. 2011;39(5):824-836.

15. Beydon L, Rouxel A, Camut N, et al. Sedative premedication before surgery-A multicentre randomized study versus placebo. Anaesth Crit Care Pain Med. 2015;34(3):165-171.
16. Maurice-Szamburski A, Auquier P, Viarre-Oreal V, et al. Effect of sedative premedication on patient experience after general anesthesia: a randomized clinical trial. JAMA. 2015;313(9):916-925.

17. Scavee V, Dehullu JP, Scavee JP, Michel I. Impact of anxiety in ambulatory superficial venous surgery: a prospective study using the HADS-A Scale. Acta Chir Belg. 2015;115(1):42-48.

18. Singh S, Maldonado Y, Taylor MA. Optimal perioperative medical management of the vascular surgery patient. Anesthes Clin. 2014;32(3):615-637.

19. Ong CK, Seymour RA, Lirk P, Merry AF. Combining paracetamol (acetaminophen) with nonsteroidal antiinflammatory drugs: a qualitative systematic review of analgesic efficacy for acute postoperative pain. Anesth Analg. 2010;110(4):1170- 1179.

20. Sen A, Erdivanli B, Ozdemir A, Kazdal H, Tugcugil E. Efficacy of continuous epidural analgesia versus total intravenous analgesia on postoperative pain control in endovascular abdominal aortic aneurysm repair: a retrospective case-control study. Bio Med Res Int. 2014; 2014:205164.

21. Major CP Jr, Greer MS, Russell WL, Roe SM. Postoperative pulmonary complications and morbidity after abdominal aneurysmectomy: a comparison of postoperative epidural versus parenteral opioid analgesia. Am Surg. 1996;62(1):45-51.

22. Karanikolas M, Aretha D, Tsolakis I, et al. Optimized perioperative analgesia reduces chronic phantom limb pain intensity, prevalence, and frequency: a prospective, randomized, clinical trial. Anesthesiology. 2011;114(5):1144-1154.

23. Katz SG, Kohl RD. Selective use of the intensive care unit after nonaortic arterial surgery. J Vasc Surg. 1996;24(2):235-239.

24. Bucknall T, Manias E, Botti M. Nurses' reassessment of postoperative pain after analgesic administration. Clin J Pain. 2007;23(1):1-7.

25. Ahmed A, Latif N, Khan R. Post-operative analgesia for major abdominal surgery and its effectiveness in a tertiary care hospital. JAnaesthesiol Clin Pharmacol. 2013;29(4):472-477.

26. Dihle A, Bjolseth G, Helseth S. The gap between saying and doing in postoperative pain management. J Clin Nurs. 2006;15(4):469-479.

27. Hartog CS, Rothaug J, Goettermann A, Zimmer A, Meissner W. Room for improvement: nurses' and physicians' views of a postoperative pain management program. Acta Anaesthesiol Scand. 2010; 54(3):277-283.

28. Triva P, Jukic M, Puljak L. Access to public healthcare services and waiting times for patients with chronic nonmalignant pain: feedback from a tertiary pain clinic. Acta Clin Croat. 2013;52(1):79-85.

29. Lesin M, Sundov ZD, Jukic M, Puljak L. Postoperative pain in complex ophthalmic surgical procedures: comparing practice with guidelines. Pain Med. 2014;15(6):1036-1042.

30. Kaji AH, Schriger D, Green S. Looking through the retrospectoscope: reducing bias in emergency medicine chart review studies. Ann Emerg Med. 2014;64(3):292-298.
Journal of Pain Research

\section{Publish your work in this journal}

The Journal of Pain Research is an international, peer reviewed, open access, online journal that welcomes laboratory and clinical findings in the fields of pain research and the prevention and management of pain. Original research, reviews, symposium reports, hypothesis formation and commentaries are all considered for publication.

\section{Dovepress}

The manuscript management system is completely online and includes a very quick and fair peer-review system, which is all easy to use. Visit http://www.dovepress.com/testimonials.php to read real quotes from published authors. 